\title{
Some results on Triple Hypergeometric functions involving Integral representations
}

\author{
Darshowkat $^{1}$, Ab. Rashid Dar ${ }^{2}$ \\ Department of Mathematics \& Statistics, Govt. Degree collage Sopore, Kashmir, India-193201 \\ Department of Mathematics \& Statistics, Govt. Degree collage Sopore, Kashmir, India-193201
}

\begin{abstract}
In this paper our aim is to prove some certain results on Triple Hypergeometric functions involving Integrals, where everyone can be represented like as Euler integral representations. Also some of results are obtained as special cases of our main results
\end{abstract}

Keywords: Generalized Hypergeometric functions, Gauss Hypergeometric functions, Picard's integral formula, triple Hypergeometric functions, Appell functions, Beta and Gamma functions.

\section{Introduction}

The function $F(a, b ; c ; z)$ in the study of second order linear differential equation with three regular singular points, there arise the function (see ref. [1], chapter 4, pp. 45) and ref. [2] defined by

(1.1) $F[a, \mathrm{~b} ; \mathrm{c} ; \mathrm{z}]=\sum_{n=0}^{\infty} \frac{(a)_{n}(b)_{n}}{(c)_{n}} \frac{z^{n}}{n !}, \mathrm{C} \neq 0,-1,-2,-3 \ldots$,

is called hypergeometric series. In term of the Pochhammmer symbol the infinite series (1.1) is evidently reduced to the elementary geometric series.

(1.2) $\sum_{n=0}^{\infty} z^{n}=1+Z+Z^{2}+Z^{3}+\cdots$

In its special case when
i) $a=c$ and $b=1$.

ii) $a=1$ and $b=c$.

Hence it is called the hypergeometric series or more precisely Gauss's hypergeometric series after the famous German mathematical Carl Friedrich Gauss (1777-1855), who in the year 1812 introduced the series into analysis and gave F-function for it. Test shows that the hypergeometric series in (1.2) when $|z|=1$ (that is on a unit circle) is:-

i) Absolutely convergent if $\operatorname{Re}(\mathrm{c}-a-\mathrm{b})>0$;

ii) Conditionally convergent if $-1<\operatorname{Re}(\mathrm{c}-a-\mathrm{b}) \leq 0, \mathrm{Z} \neq 0$;

iii) Divergent if $\operatorname{Re}(\mathrm{c}-a-b) \leq-1$.

A simple integral representation of the hypergeometric function (see ref. [3]) can be obtained by using the definition of the Beta function as follow we know that if ' $n$ ' is a non-negative integer.

$$
\begin{aligned}
& \frac{(b)_{n}}{(c)_{n}}=\frac{\Gamma(b+n) \Gamma(c)}{\Gamma(b) \Gamma(c+n)} \text { by using (1.4.2) }(\lambda)_{n}=\frac{\Gamma(\lambda+\mathrm{n})}{\Gamma(\lambda)} \text {. (1.5) } \frac{(b)_{n}}{(c)_{n}} \\
& =\frac{\Gamma(c)}{\Gamma(b) \Gamma(c-b)} \frac{\Gamma(b+n) \Gamma(c-b)}{\Gamma(c+n)} .
\end{aligned}
$$

(1.6) using $\mathrm{B}(\alpha, \beta)=\frac{\Gamma(\alpha) \Gamma(\beta)}{\Gamma(\alpha+\beta)}$.

(1.7) $\frac{(\mathrm{b})_{\mathrm{n}}}{(\mathrm{c})_{\mathrm{n}}}=\frac{\Gamma(\mathrm{c})}{\Gamma(\mathrm{b}) \Gamma(\mathrm{c}-\mathrm{b})} \mathrm{B}(\mathrm{b}+\mathrm{n}, \mathrm{c}-\mathrm{n})$.

(1.8) $\frac{(b)_{n}}{(c)_{n}}=\frac{\Gamma(c)}{\Gamma(b) \Gamma(c-b)} \int_{0}^{1} t^{b+n-1}(1-t)^{c-b-1} d t$.

Where $\operatorname{Re}(c)>\operatorname{Re}(b)>0$. We also know by the binomial theorem that if $|Z|<1$, then

$$
\begin{aligned}
&(1-\mathrm{z})^{-a}= 1+a \mathrm{z}+\frac{a(a+1)}{2 !} \cdot z^{2}+\frac{a(a+1)(a+2)}{3 !} \cdot z^{3}+\cdots \\
&(1-\mathrm{z})^{-a}=\sum_{i=0}^{n} \frac{(a)_{n} z^{n}}{n !}
\end{aligned}
$$

Hence using (1.8) and (1.10), we may write 
$(1.11) F(a, \mathrm{~b} ; \mathrm{c} ; \mathrm{z})=\sum_{n=0}^{\infty} \frac{(\alpha)_{n}(b)_{n}}{(c)_{n} n !} z^{n}$.

(1.13) $F(a, b ; c ; z)$

$$
F(a, b ; c ; z)=\frac{\Gamma(c)}{\Gamma(b) \Gamma(c-b)} \sum_{n=0}^{\infty} \frac{(a)_{n}}{n !} z^{n} \int_{0}^{1} t^{\mathrm{b}+\mathrm{n}-1}(1-t)^{\mathrm{c}-b-1} d t .
$$

$$
\begin{aligned}
& =\frac{\Gamma(c)}{\Gamma(b) \Gamma(c-b)} \int_{0}^{1} t^{\mathrm{b}-1}(1-t)^{\mathrm{c}-b-1} \cdot\left\{\sum_{n=0}^{\infty} \frac{(a)_{n}}{n !}(z t) \quad n\right\} d t .(1.14) \quad F(a, b ; c ; z) \\
& =\frac{\Gamma(c)}{\Gamma(b) \Gamma(c-b)} \int_{0}^{1} t^{\mathrm{b}-1}(1-t)^{\mathrm{c}-b-1}(1-\mathrm{zt})^{-a} d t .
\end{aligned}
$$

Thus if $|z|<1$ and $\operatorname{Re}(c)>\operatorname{Re}(b)>0$ then

$$
F(a, \mathrm{~b} ; \mathrm{c} ; \mathrm{z})=\frac{\Gamma(c)}{\Gamma(b) \Gamma(c-b)} \int_{0}^{1} t^{\mathrm{b}-1}(1-t)^{\mathrm{c}-b-1}(1-\mathrm{zt})^{-a} d \mathrm{t} .
$$

We have seen (ref. [3]) that the hypergeometric series with $z=1$ and $C$ neither zero nor a negative integer is absolutely convergent if $\operatorname{Re}(c-a-b)>0$ taking $\operatorname{Re}(c-a-b) \geq 2 \delta>0$ and comparing with the series of positive constant $\sum\left(\begin{array}{ll}1+n & 1+\delta\end{array}\right)$. We conclude in the same manner as in previous definition and using the Weierstrass M-test, that the series $F(a, b ; c ; 1)$ is uniformly convergent in the region $\operatorname{Re}(c-a-b)>0$.

Hence the function $F(a, b ; c ; 1)$ is an analytic function of $a, c, z$ if $c$ is neither zero nor a negative integer and $\operatorname{Re}(c-a-b)>0$.We then have (see ref. [3])

(1.16) $F(a, \mathrm{~b} ; \mathrm{c} ; 1)=\sum_{n=0}^{\infty} \frac{(\alpha)_{n}(b)_{n}}{(c)_{n}} \frac{z^{n}}{n !}$.

Let us also impose the condition that $\operatorname{Re}(c)>\operatorname{Re}(b)>0$. Then using (1.15) we have

$$
\begin{aligned}
& F(a, b ; c ; 1)=\frac{\Gamma(c)}{\Gamma(b) \Gamma(c-b)} \int_{0}^{1} t^{\mathrm{b}-1}(1-t)^{\mathrm{c}-b-a-1} d \mathrm{t} .(1.18) F(a, b ; c ; 1) \\
& \quad=\frac{\Gamma(c)}{\Gamma(b) \Gamma(c-b)} \cdot \frac{\Gamma(b) \Gamma(\mathrm{c}-b-a)}{\Gamma(c-b)} .(1.19) \quad F(a, b ; c ; 1)=\frac{\Gamma(c)}{\Gamma(c-a)} \frac{\Gamma(\mathrm{c}-b-a)}{\Gamma(c-b)} .
\end{aligned}
$$

Thus we have proved that if $\operatorname{Re}(c-a-b)>0, \operatorname{Re}(c)>\operatorname{Re}(b)>0$ and $C$ is neither zero nor a negative integer, then

$$
\text { (1.20) } F(a, b ; c ; 1)=\frac{\Gamma(c)}{\Gamma(c-a)} \frac{\Gamma(c-b-a)}{\Gamma(c-b)} \text {. }
$$

By analytic continuation of members of (1.20). We further conclude that theresult holds even without the condition $\operatorname{Re}(c)>\operatorname{Re}(b)>0$. The result (1.20) is often called Gauss's theorem if we take $a=-n$ in (1.20) i.e. make ' $a$ ' is a negative integer; we get the following result which is known as Vandermonde's theorem.

$(1.21) F(-n, b ; c ; 1)=\frac{\Gamma(c)}{\Gamma(c+n)} \frac{\Gamma(c-b+n)}{\Gamma(c-b)}=\frac{(c-b)_{n}}{(c)_{n}}$.

Again if we put $z=-1$ and $c=b-a+1$ in (1.15) we get

$$
\begin{aligned}
\mathrm{F}(a, \mathrm{~b} ; \mathrm{b}-a+1 ;-1) & \\
& =\frac{\Gamma(\mathrm{b}-a+1)}{\Gamma(b) \Gamma(1-a)} \int_{0}^{1} t^{\mathrm{b}-1}(1-\mathrm{t})^{-a}(1+\mathrm{t})^{-a} d \mathrm{t} \\
& =\frac{\Gamma(\mathrm{b}-a+1)}{\Gamma(b) \Gamma(1-a)} \int_{0}^{1} t^{\mathrm{b}-1}\left(1-t^{2}\right)^{-a} d t,
\end{aligned}
$$

Lastly in the theory of Hypergeometric functions of a single variable has stimulated the development of a corresponding theory in two and more variables. In $1880 \mathrm{p}$. Appell considered the product of two Gauss functions viz.

$$
{ }_{2} F_{1}(a, b ; c ; x)_{2} F_{1}\left(a^{\prime}, b^{\prime} ; c^{\prime} ; y\right)=\sum_{m, n=0}^{\infty} \frac{(a)_{m}\left(a^{\prime}\right)_{n}(b)_{m}\left(b^{\prime}\right)_{n}}{(c)_{m}\left(c^{\prime}\right)_{n}} \frac{x^{m}}{m !} \frac{y^{n}}{n !}
$$

We are led to five distinct possibilities of getting new functions .One such possibility is

$$
\sum_{m, n=0}^{\infty} \frac{(a)_{m+n}(b)_{m+n}}{(c)_{m+n}} \frac{x^{m}}{m !} \frac{y^{n}}{n !}
$$


Thus the reaming four possibility lead to the four Appell function of two variables which are defined as $F_{1}, F_{2}, F_{3}, F_{4}$ see ref. [1]. Also we noticed one of additional complete triple hypergeometric function out of three complete functions that is $H_{A}$ which is defined as follows see ref. [4, p. 43, eq. 1.5 (11) to 1.5 (1)]

$(1.25) H_{A}\left(a_{1}, a_{2}, a_{3} ; c_{1}, c_{2} ; x, y, z\right)$

$$
=\sum_{m, n, p=0}^{\infty} \frac{\left(a_{1}\right)_{\mathrm{m}+\mathrm{p}}\left(a_{2}\right)_{\mathrm{m}+\mathrm{n}}\left(a_{3}\right)_{\mathrm{n}+\mathrm{p}}}{\left(\mathrm{c}_{1}\right)_{\mathrm{m}}\left(\mathrm{c}_{2}\right)_{\mathrm{n}+\mathrm{p}}} \frac{x^{m}}{m !} \cdot \frac{y^{n}}{n !} \cdot \frac{z^{p}}{p !}
$$

$(|x|=: t<1 ;|y|=: s<1 ;|z|=: t<(1-t)(1-s))$;

Where with $\mathbb{C}$ and $\mathbb{Z}_{0}^{-}$denoting the set of complex numbers and the set of non -positive integers respectively $(\lambda) n$ is the Pochhammmer symbol defined (for $\lambda \varepsilon c$ )

$$
(1.26)(\lambda)_{n}:=\frac{\Gamma(\lambda+n)}{\Gamma(\lambda)}=\left\{\begin{array}{c}
1 \\
\lambda(\lambda+1) \ldots(\lambda+n-1), \text { if }(n \varepsilon N:=\{1,2,3 \ldots\}) .
\end{array}\right.
$$

$\Gamma(z)$ being the well known Gamma function, systematically by Srivastava's Karlsson [5,chapter 3]. The above stated three dimensional regions of convergence of the triple hypergeometric series (1.25) for $H_{A}$ respt. Were given by Srivastava [6] (see also Srivastava and Karlsson [4, section 3.4]).

$$
\begin{aligned}
& H_{A}\left(\alpha, \beta_{1}, \beta_{2} ; \gamma_{1}, \gamma_{2} ; x, y, z\right)=\frac{\Gamma\left(\gamma_{1}\right) \Gamma\left(\gamma_{2}\right)}{\Gamma\left(\gamma_{1}\right) \Gamma\left(\gamma_{2}\right) \Gamma\left(\gamma_{1}-\beta_{1}\right) \Gamma\left(\gamma_{2}-\beta_{2}\right)} \\
& \quad \cdot \int_{0}^{1} \int_{0}^{1} \xi^{\beta_{1}-1} n^{\beta_{2}-1}(1-\xi)^{\gamma_{1}-\beta_{1}-1}(1-n)^{\gamma_{2}-\beta_{2}-1}(1-y n)^{-\beta_{1}}(1-x \xi-z n)^{-\alpha} \\
& \quad \cdot\left(1-\frac{x y \xi \eta}{(1-y \eta)(1-x \xi-z \eta)}\right) d \xi d \eta, \\
& \left(\mathfrak{R}\left(\gamma_{1}\right)>\Re\left(\beta_{1}\right)>0 ;\left(\mathfrak{R}\left(\gamma_{2}\right)>\Re\left(\beta_{2}\right)>0\right) ;\right.
\end{aligned}
$$

Here in this chapter, our aim is to investigate some further integral representation for triple hypergeometric functions of $H_{A}$

\section{Main Results}

See (ref. [1]),Each of the following results on triple hypergeometric functions involving integral representation for $H_{A}$ holds true

(2.1)Results 1: $H_{A}\left(a_{1}, a_{2}, a_{3} ; c_{1}, c_{2} ; x, y, z\right)$

$$
\begin{aligned}
& =\frac{\Gamma\left(c_{2}\right)}{\Gamma\left(a_{3}\right) \Gamma\left(c_{2}-a_{3}\right)} \int_{0}^{1} \xi^{a_{3}-1}(1-\xi)^{c_{2}-a_{3}-1}(1-y \xi)^{-a_{2}} \\
& .(1-z \xi)^{-a_{1}}{ }_{2} F_{1}\left(a_{1}, a_{2} ; c_{1} ; \frac{x}{(1-y \xi)(1-z \xi)}\right) d \xi \text {, } \\
& \left(\mathfrak{R}\left(c_{2}\right)>\mathfrak{R}\left(a_{3}\right)>0\right) \text {; } \\
& \text { (2.2)Results 2: } H_{A}\left(a_{1}, a_{2}, a_{3} ; c_{1}, c_{2} ; x, y, z\right) \\
& =\frac{\Gamma\left(c_{2}\right) \Gamma(1+\lambda)^{a_{3}}}{\Gamma\left(a_{3}\right) \Gamma\left(c_{2}-a_{3}\right)} \int_{0}^{1} \xi^{a_{3}-1}(1-\xi)^{c_{2}-a_{3}-1}(1+\lambda \xi)^{a_{1}+a_{2}-c_{2}} \\
& .[1+\lambda \xi-(1+\lambda) \xi y]^{-a_{2}}[1+\lambda \xi-(1+\lambda) \xi z]^{-a_{1}} \\
& \left(\mathfrak{R}\left(c_{2}\right)>\mathfrak{R}\left(a_{3}\right)>0 ; \lambda>-1\right) ; \\
& \cdot{ }_{2} F_{1}\left(a_{1}, a_{2} ; c_{1} ; \frac{x(1+\lambda \xi)^{2}}{[1+\lambda \xi-(1+\lambda) \xi y][1+\lambda \xi-(1+\lambda) \xi z]}\right) d \xi,
\end{aligned}
$$

(2.3)Results3: $H_{A}\left(a_{1}, a_{2}, a_{3} ; c_{1}, c_{2} ; x, y, z\right)$

$$
\begin{aligned}
& \quad=\frac{\Gamma\left(c_{2}\right)}{\Gamma\left(a_{3}\right) \Gamma\left(c_{2}-a_{3}\right)} \frac{(\beta-\gamma)^{a_{3}}(\alpha-\gamma)^{c_{2}-a_{3}}}{(\beta-\alpha)^{c_{2-a_{1}-a_{2}-1}}} \\
& .[(\beta-\alpha)(\xi-\gamma)-(\beta-\gamma)(\xi-\alpha) y]^{-a_{1}}+a_{2}-c_{2} \\
& .[(\beta-\alpha)(\xi-\gamma)-(\beta-\gamma)(\xi-\alpha) z]^{-a_{1}} \\
& \left.{ }_{2} F_{1}\left(a_{1}, a_{2} ; c_{1} ; \sigma x\right) d \xi, \quad \cdot \xi\right)_{\alpha}^{c_{2} a_{3}-1}(\xi-\alpha)^{a_{3}-1}(\xi \\
& \left(\mathfrak{R}\left(c_{2}\right)>\mathfrak{R}\left(a_{3}\right)>0 ; \gamma<\alpha<\beta\right) .
\end{aligned}
$$

Where

$$
\sigma:=\frac{(\beta-\alpha)^{2}(\xi-\gamma)^{2}}{[(\beta-\alpha)(\xi-\gamma)-(\beta-\gamma)(\xi-\alpha) y][(\beta-\alpha)(\xi-\gamma)-(\beta-\gamma)(\xi-\alpha) z]} ;
$$

(2.4)Results4: $H_{A}\left(a_{1}, a_{2}, a_{3} ; c_{1}, c_{2} ; x, y, z\right)$ 


$$
\begin{aligned}
& \quad=\frac{\Gamma\left(c_{2}\right)}{\Gamma\left(a_{3}\right) \Gamma\left(c_{2}-a_{3}\right)} \int_{0}^{\infty} \xi^{a_{3}-1}(1+\xi)^{a_{1}+a_{2}-c_{2}} \\
& { }_{2} F_{1}\left(a_{1}, a_{2} ; c_{1} ; \frac{x(\mathbf{1}+\xi)^{2}}{(1+\xi-\mathbf{y} \xi)(\mathbf{1}+\xi-\mathbf{z} \xi)}\right) d \xi, \\
& \left(\Re\left(c_{2}\right)>\Re\left(a_{3}\right)>0\right) ;
\end{aligned}
$$

(2.5) Results5: $H_{A}\left(a_{1}, a_{2}, a_{3} ; c_{1}, c_{2} ; x, y, z\right)$

$$
\begin{gathered}
=\frac{2 \Gamma\left(c_{2}\right)}{\Gamma\left(a_{3}\right) \Gamma\left(c_{2}-a_{3}\right)} \int_{0}^{\frac{\pi}{2}}\left(\sin ^{2} \xi\right)^{a_{3}-\frac{1}{2}}\left(\cos ^{2} \xi\right)^{c_{2}-a_{3}-\frac{1}{2}} \\
\cdot\left(1-\operatorname{ysin}^{2} \xi\right)^{-a_{2}} \cdot\left(1-z \sin ^{2} \xi\right)^{-a_{1}} \\
\quad{ }_{2} F_{1}\left(a_{1}, a_{2} ; c_{1} ; \frac{x}{\left(1-y \sin ^{2} \xi\right)\left(1-\operatorname{zsin}^{2} \xi\right)}\right) d \xi,
\end{gathered}
$$

$\left(\Re\left(c_{2}\right)>\Re\left(a_{3}\right)>0\right)$.

Here ${ }_{2} \mathrm{~F}_{1}$ denotes the well- known gauss hypergeometric function defined by $(2.6){ }_{2} F_{1}(\mathrm{a}, \mathrm{b}, \mathrm{c} ; \mathrm{z})$

$$
\begin{aligned}
& \quad=\sum_{n=0}^{\infty} \frac{(a)_{n}(b)_{n}}{(c)_{n}} \frac{z^{n}}{n !} ; \quad \mathrm{c} \in \mathbb{C} \backslash \mathbb{Z}_{0}^{-}:|\mathrm{z}|<1 ;|\mathrm{z}|=1(\mathrm{z} \neq-1) \\
& \text { and } \Re(\mathrm{c}-a-\mathrm{b})>0 ; z=-1 \text { and } \Re(\mathrm{c}-a-\mathrm{b})>-1
\end{aligned}
$$

\section{Proof Of Main Results}

PROOF: The integral representation (2.1) was derived by Srivastavas himself [7, p. 100] as an intermediate result in his demonstration of the integral representation (1.5) [7, p. 100, eq.(3.3)]. In fact Srivastavas derivation of (2.1) involved writing the triple hypergeometric series in (1.15) as a single series of the Appell function $F_{1}$ as follows:

(3.1) $H_{A_{1}}\left(a_{1}, a_{2}, a_{3} ; c_{1}, c_{2} ; x, y, z\right)$

$$
=\sum_{m=0}^{\infty} \frac{\left(a_{1}\right)_{\mathrm{m}}\left(a_{2}\right)_{\mathrm{m}}}{\left(\mathrm{c}_{1}\right)_{\mathrm{m}}} \mathrm{F}_{1}\left[a_{3}, a_{2}+\mathrm{m}, a_{1}+\mathrm{m} ; c_{2} ; \mathrm{y}, \mathrm{z}\right] \frac{x^{m}}{m !}
$$

And then applying Picard's integral formula [4, p.29, eq.(4)]

(3.2) $F_{1}\left[\alpha, \beta, \beta^{\prime} ; \gamma ; x, y\right]$

$$
\begin{gathered}
=\frac{\Gamma(\gamma)}{\Gamma(\alpha) \Gamma(\gamma-\alpha)} \int_{0}^{1} \tau^{\alpha-1}(1-\tau)^{\gamma-\alpha-1}(1-x t)^{-\beta}(1-y t)^{\beta^{\prime}} d \tau, \\
\left(\mathrm{R}(\gamma)>R(\alpha)>0 ; \gamma \in \mathbb{C} \backslash \mathbb{Z}_{0}^{-}\right) .
\end{gathered}
$$

To each term on the right-hand side of (3.1). The transition from (2.1) to Srivastavas Final result (1.5) was made by appealing to the following classical result (see, for Details, [7, pp.99-100])

(3.3) ${ }_{2} \mathrm{~F}_{1}(\alpha, \beta ; \gamma ; \mathrm{z})$

$$
=\frac{\Gamma(\gamma)}{\Gamma(\alpha) \Gamma(\gamma-\alpha)} \int_{0}^{1} \tau^{\alpha-1}(1-\tau)^{\gamma-\alpha-1}(1-z t)^{-\beta} d \tau,
$$

$$
\left(\mathrm{R}(\gamma)>R(\alpha)>0 ; \gamma \in \mathbb{C} \backslash \mathbb{Z}_{0}^{-}\right)
$$

The assertions (2.1) to (2.5) of main results would follow from Srivastava's result uponSetting of each
i) $\tau=\xi$ and $(0,1) \rightarrow(0,1)$
ii) $t=((\beta-\gamma)(\xi-\alpha)) /((\beta-\alpha)(\xi-\gamma))$ and $(0,1) \rightarrow(\alpha, \beta)$
iii) $t=\frac{(1+\lambda) \xi}{1+\lambda \xi} ;(0,1) \rightarrow(0,1)$
iv) $\quad \xi \rightarrow \frac{\xi}{1-\xi} \quad d \xi \rightarrow \frac{d \xi}{(1-\xi)^{2}} \quad$ and $\quad(0,1) \rightarrow(0, \infty)$
v) $\quad \xi \rightarrow \sin ^{2} \xi, d \xi \rightarrow 2 \sin \xi \cos \xi d \xi ;(0,1) \rightarrow\left(0, \frac{\pi}{2}\right)$,respectively. 
Each of the integral representations (2.1) to (2.5) can also be proved directly by expressing the series definition of the involved hypergeometric functions ${ }_{2} \mathrm{~F}_{1}$ in each integrand and changing the order of the integral sign and the summation, and finally using one or the other of the following well- known relationships between the Beta functions $\mathrm{B}(\alpha, \beta)$, the gamma function $\Gamma(z)$ and their various associated Eulerian integral (see, for example [8, p.26 and pp. 86, problem])

$$
\mathrm{B}(\alpha, \beta)=\left\{\begin{array}{cl}
\int_{0}^{1} t^{\alpha-1}(1-t)^{\beta-1} \mathrm{dt}, & (\min \{\mathfrak{R}(\alpha)>0, \mathfrak{R}(\beta)\}>0) ; \\
\frac{\Gamma(\alpha) \Gamma(\beta)}{\Gamma(\alpha+\beta)}, & \left(\alpha, \beta \in \mathbb{C} \backslash \mathbb{Z}_{0}^{-}\right)
\end{array}\right.
$$

$\mathrm{B}(\alpha, \beta)=2 \int_{0}^{\pi / 2}(\sin \theta)^{2 \alpha-1}(\cos \theta)^{2 \beta-1} d \theta=\int_{0}^{\infty} \frac{r^{\alpha-1}}{(1+r)^{\alpha+\beta}} d r$,

$$
\begin{aligned}
& \mathrm{B}(\alpha, \beta)= \frac{(b-c)^{\alpha}(a-c)^{\beta}}{(b-a)^{\alpha+\beta-1}} \int_{\mathrm{a}}^{\mathrm{b}} \frac{(t-a)^{\alpha-1}(b-t)^{\beta-1}}{(t-c)^{\alpha+\beta}} \mathrm{dt} \\
&=(1+\lambda)^{\alpha} \int_{0}^{1} \frac{\mathrm{t}^{\alpha-1}(1-t)^{\beta-1}}{(1+\lambda \mathrm{t})^{\alpha+\beta}} \mathrm{dt}, \quad \lambda>-1, \\
&(\min \{\mathfrak{R}(\alpha)>0, \Re(\beta)\}>0)
\end{aligned}
$$

\section{Special Cases}

Some functions, which believed to be new, can be established as special cases as follows.

In (2.1) to (2.5) choosing $a_{3}=0$, we get following results

$$
H_{A}\left(a_{1}, a_{2} ; c_{1}, c_{2} ; x, y, z\right)=\int_{0}^{1} \xi^{-1}(1-\xi)^{c_{2}--1}(1-y \xi)^{-a_{2}}(1-z \xi)^{-a_{1}}
$$

${ }_{2} F_{1}\left(a_{1}, a_{2} ; c_{1} ; \frac{x}{(1-y \xi)(1-z \xi)}\right) d \xi$,

$\left(\mathfrak{R}\left(c_{2}\right)>0\right)$;

$$
H_{A}\left(a_{1}, a_{2} ; c_{1}, c_{2} ; x, y, z\right)=\int_{0}^{1} \xi^{-1}(1-\xi)^{c_{2}--1}(1+\lambda \xi)^{a_{1}+a_{2}-c_{2}}
$$

.$[1+\lambda \xi-(1+\lambda) \xi y]^{-a_{2}}[1+\lambda \xi-(1+\lambda) \xi z]^{-a_{1}}$

$\left(\Re\left(c_{2}\right)>0 ; \lambda>-1\right)$

$$
\cdot{ }_{2} F_{1}\left(a_{1}, a_{2} ; c_{1} ; \frac{x(1+\lambda \xi)^{2}}{[1+\lambda \xi-(1+\lambda) \xi y][1+\lambda \xi-(1+\lambda) \xi z]}\right) d \xi,
$$

$$
\begin{aligned}
& \text { (4.3) } H_{A}\left(a_{1}, a_{2} ; c_{1}, c_{2} ; x, y, z\right)=\frac{(\alpha-\gamma)^{c_{2}}}{(\beta-\alpha)^{c_{2}-a_{1}-a_{2}}} \int_{\alpha}^{\beta}(\beta-\xi)^{c_{2}-1}(\xi-\alpha)^{-1}(\xi-\gamma)^{a_{1}+a_{2}-c_{2}} \\
& .[(\beta-\alpha)(\xi-\gamma)-(\beta-\gamma)(\xi-\alpha) y]^{-a_{2}}
\end{aligned}
$$

${ }_{2} F_{1}\left(a_{1}, a_{2} ; c_{1} ; \sigma x\right) d \xi$,

$$
\text { . }[(\beta-\alpha)(\xi-\gamma)-(\beta-\gamma)(\xi-\alpha) z]^{-a_{1}}
$$

$\left(\mathfrak{R}\left(c_{2}\right)>0 ; \gamma<\alpha<\beta\right)$.

Where

$$
\sigma:=\frac{(\beta-\alpha)^{2}(\xi-\gamma)^{2}}{[(\beta-\alpha)(\xi-\gamma)-(\beta-\gamma)(\xi-\alpha) y][(\beta-\alpha)(\xi-\gamma)-(\beta-\gamma)(\xi-\alpha) z]}
$$

$$
H_{A}\left(a_{1}, a_{2} ; c_{1}, c_{2} ; x, y, z\right)=\int_{0}^{\infty} \xi^{-1}(1+\xi)^{a_{1}+a_{2}-c_{2}}
$$

$$
{ }_{2} F_{1}\left(a_{1}, a_{2} ; c_{1} ; \frac{x(\mathbf{1}+\xi)^{2}}{(1+\xi-\mathbf{y} \xi)(1+\xi-\mathbf{z} \xi)}\right) d \xi,
$$

$$
\cdot(1+\xi-y \xi)^{-a_{2}}(1+\xi-z \xi)^{-a_{1}}
$$

$\left(\Re\left(c_{2}\right)>0\right)$;

$$
\begin{aligned}
& H_{A}\left(a_{1}, a_{2} ; c_{1}, c_{2} ; x, y, z\right)=2 \int_{0}^{\frac{\pi}{2}}\left(\sin ^{2} \xi\right)^{-\frac{1}{2}}\left(\cos ^{2} \xi\right)^{c_{2}--\frac{1}{2}} \\
& .\left(1-y \sin ^{2} \xi\right)^{-a_{2}} \cdot\left(1-z \sin ^{2} \xi\right)^{-a_{1}}
\end{aligned}
$$


$\left(\mathfrak{R}\left(c_{2}\right)>0\right)$

$$
\cdot{ }_{2} F_{1}\left(a_{1}, a_{2} ; \mathrm{c}_{1} ; \frac{x}{\left(1-y \sin ^{2} \xi\right)\left(1-\mathrm{zsin}^{2} \xi\right)}\right) d \xi
$$

Our motive is to obtain other special cases from the main results

\section{Proof Of Special Cases}

Proof of (4.1) to (4.5) is much akin to that of main results (2.1) to (2.5), which we have already presented in a reasonably detailed manner.

\section{References}

[1] H. M. Srivastava's and H. L. Manocha (1984), A treatise on generating functions. Publ. Ellis Harwood limited, Co. St., Chrichester west Sussex, po191Ed, England.

[2] E. D. Rainville (1960), Special function. Macmillan, Newyork, Reprint by cheses publ. Co., Bronx, Newyork, 1971.

[3] N. Saran, S. D. Sharma and T. N. Trivedi, Special functions. Publ. Pragati Prakashan, New Market, Meerut-250 001

[4] H. M. Srivastava (1967),Some integrals representing triple hypergeometric functions. Rend. Circ. Mat. Palermo (Ser. 2), 16, 99115 .

[5] G. Lauricella, (1893), Sulle funzioni iper-geometriche a piu variabili.Rend. Cire. Mat. Palermo. 7, 111-158.

[6] A. Hasanov, and H. M, Srivastava, (2007), Decomposition formulas associated with the Lauricella multivariable hypergeometric function. comput. Math. App., 53. 1119- 1128

[7]A. Hasanov, and H. M, Srivastava, (2006), Decomposition formulas associated with the Lauricella functions $F_{A}^{(r)}$ and other multivariable hypergeometric function. Comput. Math. App., 19. 113- 121.

[8] A. HasanovH. M. Srivastava, and M. Turaev(2006), Decomposition formulas for some triple hypergeometric function. J. Math. Anal. Apple. 324, 955- 969. 